\title{
Excessive gestational weight gain and gestational diabetes: importance of the first weeks of pregnancy
}

\author{
Julie Robitaille ${ }^{1}$
}

Received: 14 July 2015 / Accepted: 22 July 2015 /Published online: 8 August 2015

(C) Springer-Verlag Berlin Heidelberg 2015

\begin{abstract}
Gestational diabetes mellitus (GDM) is associated with many adverse neonatal and maternal outcomes and prevention of this condition is considered a key strategy for breaking the intergenerational cycle of obesity and diabetes. Whether prevention of excessive gestational weight gain in the first weeks of pregnancy is associated with a reduced risk for GDM is currently unclear. In this issue of Diabetologia, Brunner et al (DOI: 10.1007/s00125-015-3686-5) address this possible association by conducting a systematic review and a meta-analysis. The results of their study provide evidence that excessive gestational weight gain prior to a GDM screening test is associated with an increased risk of GDM compared with non-excessive gestational weight gain. These results emphasise the need for appropriate preconception care and for better prevention of early excessive gestational weight gain and GDM.
\end{abstract}

Keywords Gestational diabetes mellitus (GDM) · Gestational weight gain · Pregnancy

\section{Abbreviations \\ GDM Gestational diabetes mellitus \\ GWG Gestational weight gain \\ IOM Institute of Medicine}

Gestational diabetes mellitus (GDM) is defined as glucose intolerance with onset or first recognition during pregnancy

Julie Robitaille

Julie.robitaille@fsaa.ulaval.ca

1 School of Nutrition, Institute of Nutrition and Functional Foods (INAF), Laval University, Quebec City, QC, Canada G1V 0A6 and is associated with many adverse neonatal and maternal outcomes [1]. Although treatment of this condition has been shown to be successful in preventing major perinatal and maternal complications [2], prevention of GDM is considered a key strategy for breaking the intergenerational cycle of obesity and diabetes [3]. Avoidance of excessive gestational weight gain (GWG) could be a potential preventative measure given that higher GWGs lead to greater fat deposition, which may impair insulin sensitivity $[4,5]$.

In 2009, the Institute of Medicine (IOM) published new guidelines for the management of GWG [6]. These revised guidelines include a specific recommendation for obese women and rates of GWG per week of pregnancy from the second trimester [6]. Data have indicated that since the publication of the revised 2009 IOM guidelines, approximately $50 \%$ of women have experienced excessive GWG [7]. Although excessive GWG has been associated with a number of perinatal and maternal outcomes, studies have failed to demonstrate a clear association between total GWG and the risk of GDM. This may be partly explained by intensive management of GDM, which has been proven to be successful in limiting GWG in late pregnancy, resulting in similar total GWG between women with a pregnancy complicated by GDM and women without GDM [8]. Given that screening for GDM usually occurs during the second trimester, depending on the criteria used, and given that excessive GWG may impair insulin sensitivity, the focus has now shifted to the study of the association between early GWG or GWG before screening for GDM and the subsequent risk for GDM. However, it remains unclear if excessive GWG in the first weeks of pregnancy is associated with an increased risk for GDM.

In this issue of Diabetologia, Brunner et al [9] investigated this possible association by conducting a systematic review of the literature and a meta-analysis. The aim of their study was to evaluate the relationship between excessive GWG prior to a 
GDM screening test according to IOM criteria and the risk of GDM. They analysed data from eight studies, which included 13,748 women and their results provide evidence that when assessed prior to a GDM screening test, excessive GWG according to IOM criteria is associated with an increased risk of GDM compared with non-excessive GWG. The pooled analysis yielded a summary OR of 1.40 (95\% CI: $1.21,1.61$, $p<0.001)$. Four studies included in this meta-analysis reported adjusted effect estimates for potential confounders such as maternal age, ethnicity, smoking, pre-gravid BMI, blood pressure, parity and history of GDM, and a sensitivity analysis based on these studies produced similar results. Finally, the authors performed a stratified analysis to assess potential effect modification of the association between excessive GWG and GDM by maternal pre-pregnancy BMI. They found that the effect of excessive GWG on the risk of GDM was similar between normal weight and overweight/obese women, which suggests a similar effect of early excessive GWG irrespective of pre-pregnancy BMI.

Among the limitations reported by the authors, the quality of the included studies, assessed using the GATE tool [10], was mentioned. The majority of studies were rated as being of medium quality, highlighting the need for large prospective cohort studies with (1) appropriate consideration of potential confounders; (2) valid measures of pre-pregnancy weight; and (3) different time-point measures of gestational weight. In addition, although the authors found low heterogeneity between included studies, screening tests for GDM were different across studies and cut-offs for defining excessive GWG lacked clarity in most of them. Indeed, most of the studies had measures of GWG at specific time-points such as each trimester or at specific weeks of pregnancy but few of them reported measures of GWG exactly at or before the GDM screening test. Finally, some studies that did assess the association between GWG and GDM but did not use IOM criteria for GWG were not included in this meta-analysis.

Despite these limitations, which are difficult to overcome given that the meta-analysis is based on the available literature, this study has important strengths and provides important information regarding the care of women of reproductive age. This analysis included a large number of participants $(n=$ 13,748). Also, unlike other studies that have investigated the association between GWG and the risk of GDM, this is the first meta-analysis to have assessed the association between early excessive GWG (rather than total GWG) and the risk for GDM. Given that some weight gain is associated with the normal physiological changes that occur during pregnancy, analysing data according to excessive GWG is relevant when investigating possible associations with the risk for GDM. Furthermore, the results of this meta-analysis emphasise the need for appropriate preconception and periconception care, particularly among women at high risk, such as those with prior GDM. The WHO has reported that preconception care has a positive impact on maternal and child outcomes, including prevention of complications during pregnancy and delivery, lower risk of type 2 diabetes later in life, and screening and management of diabetes in pregnancy [11]. Nevertheless, the proportion of unplanned pregnancies, currently estimated to be $40 \%$ [12], is high, which has a huge impact on the delivery of preconception and periconception care for a large proportion of women. In addition, it has been shown that women reported not being advised on how much weight to gain during pregnancy. Moreover, studies have shown that a large proportion of clinicians lack knowledge about GWG guidelines [13], suggesting that even if women can be reached before entering a pregnancy or in the first weeks of their pregnancy, clinicians need to pay more attention to appropriate weight gain during pregnancy.

The results of this study are also of great importance given that there is emerging evidence that higher GWG in the first trimester of pregnancy is associated with childhood overweight/obesity [14], as well as with postpartum weight retention and maternal cardiometabolic risk [15].

Now, what should be done? Interventions to reduce the risk of GDM often begin after the first trimester and results have been inconclusive [16]. Indeed, in a meta-analysis of 11 randomised controlled trials involving 3,744 women and their babies, there was no clear difference in the risk of developing GDM (average RR of $0.92,95 \%$ CI: $0.68,1.2$ ) between women receiving a diet and exercise intervention and those receiving standard care [16]. According to the authors of the metaanalysis, the ability to draw firm conclusions was limited by variations in the quality of trials, the characteristics of the interventions and populations assessed, and the outcome definitions between trials [16]. The timing of the beginning of the intervention should also be included as a key limitation of most of these studies. The majority of the interventions began around the end of the first trimester or later. In addition, many trials are currently under way and may provide further evidence regarding the effect of lifestyle intervention on the risk of GDM provided they address these limitations. Nevertheless, interventions including diet, exercise, or both, reduced the risk of excessive GWG by an average of $20 \%$ according to a recent meta-analysis [17]. Therefore, there is an urgent need for randomised controlled trials targeting women at high risk before they become pregnant, with the aim of preventing the development of GDM.

In conclusion, results of this timely study highlight the need for preconception care, for adequate communication of appropriate GWG by healthcare providers and for better prevention of early excessive GWG and GDM.

Duality of interest The author declares that there is no duality of interest associated with this manuscript.

Contribution statement The author was the sole contributor to this paper. 


\section{References}

1. Canadian Diabetes Association (2013) Canadian Diabetes Association 2013 clinical practice guidelines for the prevention and management of diabetes in Canada. Can J Diabetes 37:S1S212

2. Landon MB, Spong CY, Thom E et al (2009) A multicenter, randomized trial of treatment for mild gestational diabetes. N Engl $\mathrm{J}$ Med 361:1339-1348

3. Ruchat SM, Hivert MF, Bouchard L (2013) Epigenetic programming of obesity and diabetes by in utero exposure to gestational diabetes mellitus. Nutr Rev 71(Suppl 1):S88-S94

4. Lewis GF, Carpentier A, Adeli K, Giacca A (2002) Disordered fat storage and mobilization in the pathogenesis of insulin resistance and type 2 diabetes. Endocr Rev 23:201-229

5. Hedderson MM, Gunderson EP, Ferrara A (2010) Gestational weight gain and risk of gestational diabetes mellitus. Obstet Gynecol 115:597-604

6. Institute of Medicine (2009) Weight gain during pregnancy: reexamining the guidelines. The National Academies Press, Washington

7. Truong YN, Yee LM, Caughey AB, Cheng YW (2015) Weight gain in pregnancy: does the Institute of Medicine have it right? Am J Obstet Gynecol 212(362):e361-e368

8. Morisset AS, Tchernof A, Dube MC, Veillette J, Weisnagel SJ, Robitaille J (2011) Weight gain measures in women with gestational diabetes mellitus. J Womens Health (Larchmt) 20:375-380

9. Brunner S, Stecher L, Ziebarth S et al (2015) Excessive gestational weight gain prior to glucose screening and the risk of gestational diabetes: a meta-analysis. Diabetologia. doi:10.1007/s00125-0153686-5

10. Jackson R, Ameratunga S, Broad J et al (2006) The GATE frame: critical appraisal with pictures. Evid Based Med 11:35-38

11. World Health Organization (2013) Meeting to develop a global consensus on preconception care to reduce maternal and childhood mortality and morbidity. WHO, Geneva

12. Singh S, Sedgh G, Hussain R (2010) Unintended pregnancy: worldwide levels, trends, and outcomes. Stud Fam Plan 41:241-250

13. Wilkinson SA, Poad D, Stapleton H (2013) Maternal overweight and obesity: a survey of clinicians' characteristics and attitudes, and their responses to their pregnant clients. BMC Pregnancy Childbirth 13:117

14. Karachaliou M, Georgiou V, Roumeliotaki T et al (2015) Association of trimester-specific gestational weight gain with fetal growth, offspring obesity, and cardiometabolic traits in early childhood. Am J Obstet Gynecol 212(502):e501-e514

15. Walter JR, Perng W, Kleinman KP, Rifas-Shiman SL, RichEdwards JW, Oken E (2015) Associations of trimester-specific gestational weight gain with maternal adiposity and systolic blood pressure at 3 and 7 years postpartum. Am J Obstet Gynecol 212: 499.e1-12

16. Bain E, Crane M, Tieu J, Han S, Crowther CA, Middleton P (2015) Diet and exercise interventions for preventing gestational diabetes mellitus. Cochrane database of systematic reviews, issue 4, Art. no.: CD010443. doi:10.1002/14651858.CD010443.pub2

17. Muktabhant B, Lawrie TA, Lumbiganon P, Laopaiboon M (2015) Diet or exercise, or both, for preventing excessive weight gain in pregnancy. Cochrane database of systematic reviews, issue 6, Art. no.: CD007145. doi:10.1002/14651858.CD007145.pub3 